\title{
¿Para qué estudiar el síndrome de burnout en el personal de enfermería en México? Precisiones metodológicas para el desarrollo de una línea de investigación
}

\section{Why Study the burnout syndrome in nursing personnel in Mexico? Critical methodologies for developing a line of investigation}

Mtra. Victoria Fernández García,• Mtra. Rosa A. Zarate Grajales,•• Mtra. Teresa Lartigue Becerra...•

\section{Resumen}

Introducción: Diversos estudios internacionales buscan determinar la contribución del estrés laboral al problema de la escasez de enfermeras y sus efectos en la calidad del paciente. En México existe poca información sobre la magnitud del estrés laboral en enfermeras y la diversidad de metodologías y criterios utilizados para medirlo dificulta la comparación en distintos contextos nacionales. A partir de la revisión de las publicaciones nacionales, en este trabajo se discuten aspectos conceptuales y metodológicos para aproximarse al estudio del SB, con la finalidad de definir una línea de investigación que corresponda a la realidad de la práctica profesional en nuestro país. Desarrollo: Se revisa el concepto central de estrés laboral, el instrumento y los aspectos críticos para determinar la prevalencia, las teorías y modelos desarrollados en el estudio del SB, así como los diferentes niveles de análisis y relaciones a estudiar dentro del modelo del clima organizacional. Conclusiones. Es necesario desarrollar líneas de investigación utilizando un instrumento que permita la comparación y estableciendo claramente las bases teóricas subyacentes en el análisis de las relaciones del SB con las diferentes variables. Asimismo, se requiere información sobre las características organizacionales de los hospitales o unidades de salud en las que se generan las prevalencias reporta-

-Proyecto PAPIIT: El presente trabajo es una revisión de la literatura que forma parte del marco conceptual del proyecto "Coeficiente paciente enfermera, síndrome de burnout, niveles de formación y su formación con indicadores de calidad del cuidado" financiado por PAPIIT IN304907 UNAM.

- Consultora del Fondo Nestlé para la Nutrición. Mtra. en Salud Pública, Tec. Asoc. BTC. Unidad de Investigación •- Maestra en Investigación y desarrollo de la Educación. Jefa de la División de Posgrado de la eneo-UNAM.

...Doctora en investigación en Psicología, Asociación Psicoanalítica Mexicana A.C.

Correspondencia: Vicky@tunsalud.org.mx

ReCiBido: 8 DE AGOSTO DE 2010.

Enviado a Correciones: 9 DE Febrero de 2010.

Aceptado: 10 de Febrero de 2010 


\section{Enfermería Universitaria}

das, así como los coeficientes pacientes/enfermera relacionadas con las mismas y preferentemente, incluir una variable de resultado en la salud del paciente, de la enfermera o de la organización De esta manera sería posible conocer la prevalencia de SB, los factores asociados a su origen, a sus efectos y posibles soluciones.

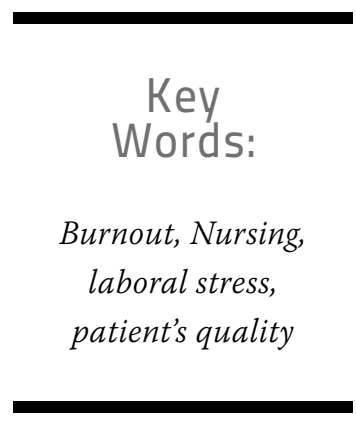

Introduction: Several international studies try to determine the contribution of laboral stress to the nursing shortage and its impact in the patient's quality. In Mexico, there is little information about the magnitude of laboral stress in nurses and the diversity of methodologies, and criteria used for assessment sets a dificulty in comparing different national contexts. At the beginning of National publications, in This paper are discussed conceptual matters and methodologies to get closse to the SB study, with the purpose of defining a line of investigation that corresponds to reality of profesional practice in our country. Development: The concept of laboral stress, the instrument and critical aspects for determining prevalence, theories and models developed in the SB study, as well as different levels of analysis and relationsships for study within the organizational model are reviewed. Conclusions: It is necessary to develop lines of investigation using an instrument that allows comparison and clearly establishes theroretical underlying basis in the analysis of SB relationships with different variables. Likewise, information is required about hospital organizational characteristics and health units in which the reported prevalences are reported, as well as related coeficients patient/nurse, and preferably to include a variable of the result in the patient and the nurse's health or that of the organization. In this way it would be posible to know the SB prevalence, the associated factors to its origin, its effects and posible solutions.

\section{INTRODUCCIÓN}

En los países desarrollados existe una gran preocupación por la escasez de enfermeras, las causas que la originan y sus efectos en la calidad de la atención y en los resultados en la salud de los pacientes. De manera particular, se busca determinar la contribución del estrés laboral a este problema. Diferentes instituciones en los Estados Unidos ${ }^{1}$ y Canadá $^{2}$ han estudiado y reconocido las posibles implicaciones del agotamiento emocional de los profesionales de enfermería. En la misma línea, el Reino Unido ${ }^{3}$ ha puesto especial interés en estudiar los am- bientes laborales y sus efectos, así como las posibles intervenciones para crear ambientes laborales positivos.

El estudio realizado por The Internacional Hospital Outcomes Study Cosortium incluyó 700 hospitales en 5 países (EEUU, Canadá, Inglaterra, Alemania y Escocia), y probó un modelo conceptual de los mecanismos por los que las características organizacionales de un hospital afectan la salud de los pacientes y las enfermeras ${ }^{4}$. Los resultados mostraron que en las jurisdicciones con deficiencias en la atención se observan también altos niveles de insatisfacción en el trabajo, el síndrome de burnout (SB) y la intención de abandonar el empleo. Este hallazgo fue especialmente marcado en contextos en los que existe una escasez de enferme$\mathrm{ras}^{4}$, y los resultados fueron consistentes con un segundo reporte del mismo estudio en el que se muestran altas proporciones de enfermeras insatisfechas con su trabajo (con excepción de Alemania). Asimismo, entre el 30 y el $40 \%$ de las enfermeras presentaron registros más altos de SB que los de las nor- 
mas recomendadas para trabajadores de la salud publicadas por los autores. $^{5}$

De esta manera, los países desarrollados con escasez de enfermeras realizan estudios dirigidos claramente al diseño de intervenciones en sus contextos específicos, con el fin de retener al personal, ofrecer mejor calidad en la atención y obtener los mejores resultados posibles en la salud de sus pacientes. En México, las investigaciones sobre el SB se centran principalmente en conocer la magnitud y algunas manifestaciones del problema, si bien diversos aspectos metodológicos dificultan el análisis de conjunto y, por tanto, el avance en el estudio del SB en el contexto nacional. En términos de políticas de servicios de salud en sus diferentes niveles de aplicación, a saber, salud pública, institucional y en las unidades de diferentes niveles de atención, las preguntas para las que se busca una respuesta son: ¿Qué información es necesaria y relevante? ¿Cuáles son las aplicaciones específicas en el contexto de la enfermería nacional e institucional?

A la luz de estas preguntas se realizó una revisión para identificar publicaciones sobre SB en enfermeras mexicanas en las bases de Medline (1 artículo) ${ }^{6}$ y Artemisa ( 4 artículos) ${ }^{6-9}$, las cuales se muestran en el cuadro 1 con sus características principales. Esta revisión da cuenta de serias limitaciones para comparar las prevalencias reportadas en cada estudio, así como las asociaciones significativas, indispensables para conocer la magnitud del problema y profundizar en los factores relevantes para la enfermería nacional. Por estas razones, el objetivo del presente trabajo es revisar los aspectos conceptuales y metodoló- gicos para aproximarse al estudio del SB, con la finalidad de definir una línea de investigación que corresponda a la realidad de la práctica profesional en nuestro país.

\section{Desarrollo \\ El estrés laboral como concepto central}

El concepto de estrés ha cambiado a lo largo de los años y existen diversas perspectivas para abordarlo. Es importante por tanto hacer una recopilación que permita comprender la manera en que se dan las relaciones de sobrecarga de trabajoestrés-SB. Retomamos para este fin el análisis de Rojas: ${ }^{10}$

Desde la perspectiva psicoanalítica, para Freud, la angustia del nacimiento se repite numerosas veces en la primera infancia ante situaciones de excesiva excitación interna o externa, por lo que, en circunstancias de peligro interno y externo, el modelo de respuesta queda grabado en la psique. En virtud de ello, Freud afirmaba que los problemas de la vida cotidiana y el ritmo intenso de vida que las sociedades imponen plantean al individuo situaciones en las que se siente amenazado, si bien la valoración de éstas es diferente para cada individuo en función de lo que cada quien reconoce como peligro y los recursos con los que cuenta para enfrentarlo. En este sentido, Rojas refiere que lo planteado por Freud como angustia y ansiedad corresponde a lo referido como estrés en la literatura médica y la psicología anglosajona.

$\mathrm{El}$ aparato psíquico, en su función de transmisor de las cargas energéticas y de mantenimiento del equilibrio interno, emprende la estructuración de los síntomas para evitar que el aparato se desborde por un conflicto en el que la angustia funciona como señal del mismo. Es así como puede comprenderse la aparición de síntomas tales como trastornos del sueño y dificultad de concentración, entre otros, como signos de un trabajo psíquico que está tratando de elaborar un conflicto interno-externo.

Otra perspectiva es la de la medicina psicosomática, que si bien se fundamentó en el psicoanálisis, consideró que los conflictos reprimidos conducían finalmente a manifestaciones orgánicas que podían tener un origen externo. Más adelante, sin embargo, Stora (citado por Rojas) planteó: "Es la estructura de la personalidad la que fragiliza una parte determinada del organismo ante la agresión interna y la que prepara el terreno para la somatización". ${ }^{10}$

El enfoque anglosajón del estrés, en los años treinta, surge en los EEUU desde el campo de la fisiología y considera que se trata de una perturbación de la homeostasis ante situaciones de frío, falta de oxígeno, descenso de la glicemia y otros. Más adelante Seyle ${ }^{11}$, considerado el padre de la teoría del estrés, y luego de algunas de sus etapas de investigación, lo definió como un conjunto de reacciones fisiológicas ante cualquier forma de estímulo nocivo, incluidas las amenazas psicológicas. A este conjunto de reacciones, Seyle le dio el nombre de síndrome general de adaptación, en el que la demanda ambiental sería el estímulo estresante. El estrés representa una respuesta del organismo a los desafíos que la persona debe enfrentar en la vida; esta respuesta consta de tres fases diferenciadas. La primera es la alarma ante la amenaza de una situación potencialmente peligro- 
sa. Si la causa que produjo la reacción de alarma no desaparece, los cambios fisiológicos que se experimentan producen una mayor resistencia en el organismo, de manera que se puede afrontar la situación, o bien, escapar de ella. Si la situación se prolonga sobreviene la tercera fase, que es de agotamiento. Cabe destacar que Seyle distinguió entre un estrés positivo (eustrés), que es la excitación que produce lo nuevo, el descubrimiento, la aventura y el reto, y el negativo, al cual llamó distrés. En años recientes se ha desarrollado una amplia investigación acerca de dos tipos de estrés negativo, el trastorno por estrés postraumático y el estrés crónico. En ambos casos, partes del cuerpo y de la mente continúan en la fase de alarma o de resistencia, y aun cuando la situación que produjo esas reacciones haya quedado atrás, el organismo reacciona más ante estímulos pasados que ante la condición presente sin que medie la intervención de la voluntad o la conciencia ${ }^{11}$.

A partir de la década de los setenta se ha ido aceptando al estrés como un aspecto inevitable de la condición humana; en paralelo, se ha observado que el afrontamiento del estrés en lo que se refiere a la adaptación establece grandes diferencias en el resultado final. También se habla del estrés socialmente inducido como factor desencadenante de padecimientos. ${ }^{11}$

\section{Prevalencia del SB: definición e instrumento}

En la historia del estudio del burnout se reconoce que el término ha sido empleado para referirse a diferentes cosas, lo cual se refleja en las revisiones realizadas por algunos autores ${ }^{12,13}$. La definición más aceptada es la de Maslach y Jackson. Maslach ${ }^{14}$, luego de 25 años de investigaciones, considera que el concepto de "quemado" en el trabajo es un síndrome psicológico que de desarrolla en respuesta a estresores crónicos en el trabajo, y las dimensiones de esta respuesta son:
a) Agotamiento abrumador;
b) Sentimientos de cinismo y distanciamiento del trabajo;
c) Sentimiento de ineficacia y carencia de logros personales.

El componente de agotamiento representa el estrés individual básico de la dimensión de burnout; el cinismo (o despersonalización) representa la dimensión interpersonal del contexto y, finalmente, el componente de ineficacia y carencia de logros representa la dimensión de auto-evaluación. La validación de la escala (Maslach Burnout Inventory) y sus dimensiones (subescalas) han sido ampliamente validadas por los autores, quienes han definido como punto de corte el último tercil de la distribución en su población estudiada.14, 15

\section{BURNOUT COMO UN TIPO DE ESTRÉS}

Desde el enfoque anglosajón, y a partir de las aportaciones de McGrath y Schulder (citados por Cordes), ${ }^{12} \mathrm{el}$ estrés se define como una condición dinámica en la que el individuo es confrontado con una oportunidad, una limitación o una demanda sobre ser/tener/hacer lo que uno desea y por lo que la resolución es percibida como incierta, pero importante para los resultados. El burnout es un aspecto distintivo del estrés que ha sido definido y estudiado primordialmente como un patrón de repuestas a estresores del trabajo.

Actualmente existe un acuerdo en cuanto a que la experiencia de estrés en el trabajo se relaciona por lo general con la calidad de vida en el mismo, incrementa la morbilidad psiquiátrica y puede contribuir a algunas formas de enfermedad física, con particular referencia a problemas músculo-esqueléticos, estrés y depresión, ${ }^{16} \mathrm{si}$ bien la revisión de Arribas $^{17}$ mostró resultados contradictorios al respecto.

Existe consenso sobre lo que se considera estrés en el trabajo. Según el Consejo Internacional de Enfermeras, éste consiste en las reacciones emocionales y físicas perjudiciales que se producen en las interacciones entre el trabajador y su entorno de trabajo, cuando las demandas del puesto superan las capacidades y recursos del propio trabajador. $^{18}$

\section{BURNOUT COMO PROCESO}

Diversos autores han planteado que el SB es un proceso. Chernis (citado por Garcés de los Fayos) ${ }^{13}$ destaca tres momentos:

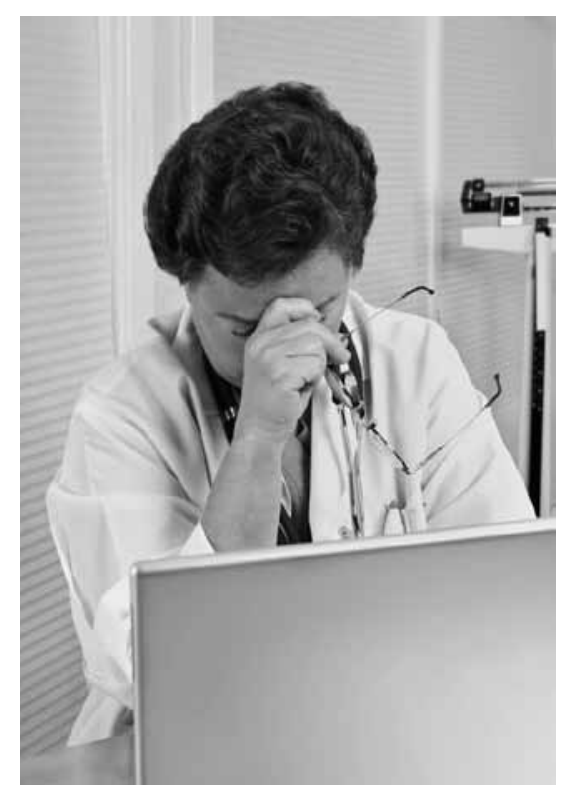


1. Desequilibrio entre las demandas en el trabajo y los recursos individuales (estrés).

2. Respuesta emocional en el corto plazo, ansiedad, tensión, fatiga y agotamiento (tensión).

3. Cambios en las actitudes y las conductas (afrontamiento defensivo).

El mismo autor cita el enfoque de Eldewich y Brodsky, en el que se proponen cuatro fases: ${ }^{19}$

1. Entusiasmo, aspiraciones elevadas, energía desbordante y sin noción de peligro.

2. Estancamiento, que surge tras no cumplirse las expectativas originales, y en el que empieza a aparecer la frustración.

3. Frustración, que consiste en el inicio de problemas emocionales, físicos y conductuales. En esta fase radica el núcleo del síndrome.

4. Apatía, que sufre el individuo y constituye el mecanismo de defensa ante la frustración.

Respecto de si es un proceso o no, la evidencia es insuficiente en estudios longitudinales para documentar las fases y su secuencia. Múltiples estudios han analizado la antigüedad en el trabajo como un predictor asociado con mayores puntajes de burnout, pero la evidencia ha sido inconsistente..$^{20}$

Si partimos de que, de acuerdo con lo referido por otros autores, ${ }^{12,}$ ${ }^{13}$ la definición más aceptada en la práctica es la de Maslach, es un hecho que también se hace referencia a la medición del SB en vinculación con instrumentos diferentes al MBI y sus variantes ${ }^{21}$. En este punto surgen las siguientes reflexiones:

- El instrumento diseñado para medir SB en trabajadores de servicios humanos

que corresponde a las

características del trabajo del

personal de enfermería es el

Maslach Burnout Inventory-

Human Service Survey (MBI-

HSS). En la literatura nacional

se observa la utilización del

MBI-General Survey

(MBI-GS), cuyo diseño no

considera al destinatario

del servicio (el usuario o

el paciente, en el caso de

enfermería), aspecto que es

fundamental en la naturaleza

del trabajo cotidiano de las

enfermeras. Otros autores

han llamado la atención sobre

este aspecto, y si bien en otros

países se aplica la MBI-GS en

enfermeras, por lo general lo

que se busca con ello es la

comparación del síndrome

con otros profesionales no

relacionados con el servicio

de ayuda a otros, ya que la

característica de este

instrumento es que mantiene

la estructura factorial a lo largo

de diversas profesiones.22, 23

- Al utilizar la definición de burnout propuesta por

Maslach, otro aspecto fundamental en este punto es la utilización de instrumentos distintos al MBI, dada la imposibilidad de comparar las prevalencias observadas ya sea en las diferentes instituciones (SSA, IMSS, ISSSTE, etc.), ámbitos (primer nivel u hospital) o regiones del país. Es por este motivo que es importante precisar qué variante del Maslach Burnout Inventory fue utilizada. Estos aspectos son fundamentales en la construcción de un panorama nacional sobre la situación del personal de enfermería.

Al mismo tiempo, también se observan estudios con insuficiente información sobre el instrumento empleado y sobre su validez y confiabilidad.

- Respecto de la prevalencia del SB en los diferentes ámbitos del país, se hace referencia a un incremento en la prevalencia en años recientes, afirmación difícil de sostener debido al incipiente desarrollo en el campo y los problemas obvios de comparabilidad antes mencionados. Es importante destacar la necesidad de estudios basales y cortes subsecuentes, o bien, de diseños longitudinales que acrediten incidencia, ya que lo cierto es que las prevalencias en el ámbito profesional siguen siendo terreno desconocido, y no existe un solo estudio con algún tipo de representatividad institucional, o por especialidad o ámbito laboral en el caso de las enfermeras mexicanas.

\section{Teorías y modelos en el estudio del burnout}

En relación con los modelos utilizados en el estudio del Síndrome de burnout, Maslach ${ }^{14}$ refiere que la mejor teoría es la de trabajadores idealistas que han experimentado burnout. La idea es que el agotamiento y el cinismo se presentan principalmente en personas que realizan un gran esfuerzo para apoyar sus idea- 
les y que constatan que sus sacrificios no son suficientes para alcanzarlos.

Una segunda teoría se refiere al burnout como resultado final de una larga exposición a estresares, es decir, el síndrome se ve antecedido por una fase de estabilidad y aparece sólo en etapas tardías de las carreras de las personas.

A la luz de estas teorías se han planteado modelos para dar respuesta a las siguientes preguntas que formula Maslach ${ }^{14}$ :

- La sobrecarga de trabajo, ¿es producto de un exceso de demanda con pocos recursos o de una monotonía?

- ¿Es la despersonalización (cinismo) la primera fase, seguida por la ineficiencia y, finalmente, el agotamiento, o éstos se desarrollan simultáneamente?

- ¿Es primero el agotamiento, y es éste el que precipita el desarrollo de la despersonalización (cinismo) y tiene como consecuencia la ineficacia?

Según la misma autora, la investigación ha establecido un vínculo secuencial entre el agotamiento y la despersonalización, pero el paso secuencial a la ineficacia es poco claro.

Por su parte, Gil-Monte y Peiró $^{24}$ plantean que las propuestas actuales de modelos elaborados desde consideraciones psicosociales pueden agruparse de la siguiente manera:

a) Modelos desarrollados en el marco de la teoría socio cognitiva del yo, caracterizados por las ideas de Bandura y plantean que el sujeto pondrá empeño en conseguir sus objetivos, según el grado de seguridad en sus propias capacidades, de esta seguridad dependerá la facilidad en conseguirlos. Los modelos desarrollados desde esta perspectiva se centran en la autoeficacia percibida o en la autoconfianza profesional como variables relevantes para el desarrollo del síndrome.

b) Modelos desde las teorías del intercambio social.

Esta perspectiva sostiene que el SB tiene su etiología fundamentalmente en las percepciones de una falta de equidad en el proceso de comparación que tiene lugar en los individuos al establecer relaciones interpersonales. En este caso se plantea que en las relaciones de intercambio que establecen las enfermeras con los pacientes o usuarios, los compañeros de trabajo, los supervisores y con la misma organización se genera una expectativa de equidad no satisfecha derivada de la percepción de un desequilibrio entre lo dado y lo recibido, lo cual da como resultado el SB.

c) Modelos desde la teoría organizacional, que parten de las disfunciones y pobreza del rol, sobrecarga de trabajo, la falta de salud organizacional, la estructura, la cultura y el clima organizacional. Estos, enfatizan el papel de los estresores en el lugar de trabajo.

Los mismos autores proponen la integración de algunos modelos, que el SB debe considerarse como una respuesta al estrés laboral per- cibido, el cual surge luego de un proceso de reevaluación cognitiva, cuando las estrategias de afrontamiento empleadas por los profesionales son ineficaces para reducir el estrés percibido. Esta propuesta supone una variable mediadora entre el estrés y sus consecuencias, en la que el bajo desempeño en el trabajo y los sentimientos agudos de agotamiento emocional se inician de manera simultánea. Las actitudes de despersonalización tienen lugar posteriormente como una estrategia de afrontamiento. ${ }^{24}$

- A la luz de los aspectos revisados y de la producción nacional, surge la reflexión sobre la necesidad de partir claramente de propuestas teóricas, necesidad que es poco reconocida en gran parte de la literatura nacional e internacional.

Sin embargo, si el objetivo es contribuir a la construcción de evidencia sobre las causas, los efectos, las relaciones entre variables y el efecto del burnout en variables de resultado tales como la salud de los pacientes, este aspecto es fundamental para dejar clara la dirección que queremos imprimir en el estudio del SB, lo que por supuesto incluye el cuidado de la correspondencia con el instrumento utilizado.

\section{Niveles de análisis en el estudio del SB}

En el campo de la investigación del SB se observa la utilización en diversos estudios de los mismos términos con diferentes connotaciones; por ejemplo, los aspectos organizacionales suelen ser referi- 
Figura 1. Resultados del clima organizacional y de la salud de la enfermera (Gershon R, 2007)

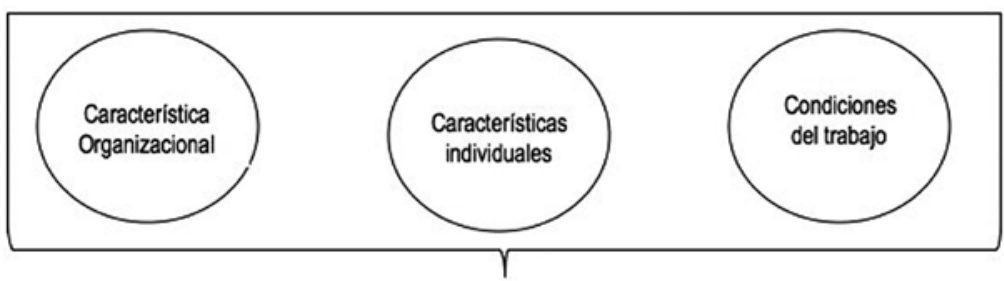

Dominios de la calidad de vida laboral

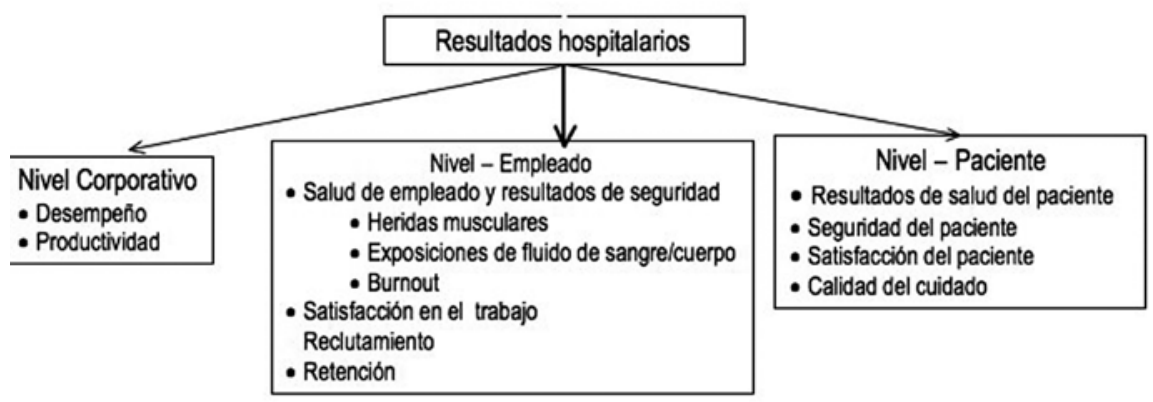

dos como condiciones de trabajo, clima organizacional o características del trabajo, por lo que Gershon et al25 sugieren agrupar los factores en tres dominios mayores: características organizacionales, características individuales y condiciones de trabajo. Los factores agrupados se muestran en el modelo construido por Beehr y et al. (citado por Gershon et al.), que puede apreciarse en la figura 1.

\section{- Características organizacio-} nales. Estas son el tamaño y el tipo de hospital (para entender la complejidad de la organización y de las tareas), el financiamiento (relacionado con la disponibilidad de los recursos), el tipo de gobierno y la estructura jerárquica (el lugar que ocupa la líder de enfermería), el tipo de cultura organizacional (entendida como principios, valores y normas subyacentes en la organización) y, finalmente, la estabilidad organizacional.
El trabajo de las enfermeras tiene lugar en organizaciones que incluyen jerarquías, reglas, recursos y una distribución del espacio, así como un contrato psicológico entre la institución y el trabajador relacionado con el ambiente gerencial. Desde esta perspectiva destaca la importancia de los valores implícitos en los procesos organizacionales y estructurales. Estos valores son parte de las relaciones emocionales y cognitivas que las personas desarrollan en su trabajo. Se ha documentado que los cambios y ajustes afectan al personal, sobre todo si el cambio ocurre en el contrato psicológico, cuando se percibe que las expectativas básicas de equidad y justicia son violadas. ${ }^{14}$

En el contexto de las políticas laborales, a las enfermeras se les exige mayores habilidades y competencias, mientras que reciben menos en términos de seguridad en el empleo y prestaciones. Se ha propuesto como hipótesis que la violación del contrato psicológico produce el burnout en virtud de la percepción de falta de reciprocidad, que es crucial para el mantenimiento del bienestar.

- Características individuales. Características demográficas, estatus socioeconómico, percepciones individuales, estresores de la vida y estrategias de enfrentamiento, ajuste persona-trabajo, apoyo social, valores y significados del trabajo, competencias para el trabajo (conocimientos y habilidades), experiencia de trabajo (puestos ocupados).

En este contexto, algunos autores han discutido sobre los factores relacionados con la experiencia de estrés de las enfermeras, debido a que los resultados en los estudios son muy variables aun en el mismo ámbito ${ }^{26,27}$. El concepto básico de estrés se relaciona con una percepción de demanda y la capacidad de respuesta: un desajuste entre ambos dispara una respuesta de estrés. Ésta dependerá de las características de la persona, su experiencia, sus mecanismos de afrontamiento y las circunstancias en las que las demandas están siendo formuladas. A este planteamiento subyace el aspecto subjetivo de la percepción del estrés. Mc Vicar ${ }^{27}$ propone considerar el estrés como un continuum en el que se pasa del estrés positivo (eustrés) a un estrés moderado o severo.

- Condiciones de trabajo. Según el modelo Beerh, éstas incluyen: clima organizacional, características y ambiente en el trabajo.

- El clima organizacional se refiere a las percepciones que 
tiene el grupo de atributos organizacionales tale como la toma de decisiones, las características de la líder de enfermería, las normas, los valores y el apoyo del (la) líder y/o supervisor(a) para la seguridad de enfermeras y pacientes.

- Características del trabajo. Incluyen al personal (cantidad, oportunidades de capacitación, oportunidades de ascenso), la programación del trabajo (relación enfermera/paciente), las demandas de trabajo (carga laboral, demandas físicas y psicológicas), la toma de decisiones y las relaciones interpersonales, los riesgos ocupacionales, los recursos (equipo, insumos), el tipo de pacientes (agudos, crónicos), el salario y las prestaciones.

- Ambiente de trabajo. Considera la ubicación de la unidad u hospital y las condiciones físicas de la construcción.

Según el modelo citado en la figura 1 , las características organizacionales e individuales y las condiciones laborales determinan la calidad de vida en el trabajo y producen diferentes tipos de resultados en el hospital en distintos niveles:

- Organización: alcance de objetivos y productividad.

- Enfermera: salud y seguridad, lesiones músculo-esqueléticas, exposición a fluidos, burnout, satisfacción en el trabajo, reclutamiento suficiente y permanencia del personal.

- Paciente: resultados en su salud y su seguridad, satisfacción y calidad del cuidado.
El modelo antes expuesto y sus componentes permiten identificar diversos factores en dominios mayores, útiles para organizar los factores que habrá que estudiar y para establecer el nivel potencial de análisis que puede realizarse; de manera particular, ubica el estudio del burnout y sus posibles relaciones.

En este contexto es posible determinar todos los estudios encaminados a identificar las fuentes de estrés en el trabajo de las enfermeras, línea de investigación que ha tenido un desarrollo amplio desde los años setenta. Revans, en 1976 (citado por McGrath et al. 1989) ${ }^{26}$, describía ya el trabajo en hospitales como estresante por la cercanía con el dolor y la muerte, así como por el hecho de realizar tareas desagradables; McGrath en su revisión documentó como principales fuentes de estrés la sobrecarga de trabajo, la dificultad con otros miembros del personal, el sistema de turnos, las relaciones con los médicos, el no poder elegir los compañeros de trabajo, la falta de reconocimiento, las fluctuaciones en la cantidad de trabajo, la desorganización del hospital, la antigüedad en la profesión y la falta de retroalimentación o descalificación.

También se ha supuesto que la atención a cierto tipo de pacientes, como los hospitalizados por razones oncológicas o en estado críti$\mathrm{Co}^{28,29}$ incrementa el estrés experimentado por las enfermeras. En diversos estudios, algunas áreas específicas de trabajo dentro del hospital se han asociado con un mayor estrés; en estas áreas, Maldonado ${ }^{30}$ refiere como principales causas de estrés los conflictos con los médicos y otros miembros del equipo de salud, así como la sobrecarga de trabajo, pero sobre todo el hecho de que las enfermeras no son consideradas en la toma de decisiones, ni su opinión es tomada en cuenta en la atención que se presta para el alivio del paciente. Destacan también la exposición a monitores y alarmas, así como las situaciones de angustia con mayor probabilidad de conflicto.

En este marco, Mc Vicar ${ }^{27}$ realizó una revisión exhaustiva de los estudios sobre estrés en el lugar de trabajo del personal de enfermería, en el periodo de 1985 a abril del 2003, e identificó seis temáticas estudiadas sobre las fuentes de estrés en el trabajo en 41 estudios publicados:.

- Carga de trabajo/personal inadecuado/presión de tiempo.

- Relación con otros miembros del personal clínico.

- Estilo de liderazgo y administración pobre, tramo de control excesivo, pobre cohesión de grupo y apoyo deficiente en la supervisión.

- Afrontamiento de las necesidades de los pacientes y sus familias, deficiencias en el diagnóstico del paciente, muerte y agonía.

- Cambio de trabajo.

- Recompensa deficiente.

Estos aspectos están relacionados con características organizacionales y personales de las enfermeras, por lo que en países desarrollados se ha considerado que el estrés laboral está teniendo un impacto en la fuerza de trabajo y han supuesto que la intensidad del estrés procedente de las fuentes ya reconocidas se ha in- 
crementado y/o que éstas contribuyen a la generación de otras fuentes adicionales. ${ }^{27}$ Por ejemplo, en estos países, estudios recientes han encontrado que la deficiencia en la recompensa y el cambio de trabajo son fuentes mayores de estrés.

Existe una producción constante de estudios con este enfoque que se orientan incluso a la identificación de soluciones. Un ejemplo es la revisión de Shirey ${ }^{31}$, en la que identificó 31 artículos (1980-2003) centrados en el estudio del estrés y el rol de las enfermeras administradoras; en esta revisión la propuesta apunta al análisis de esta figura profesional, debido a que en la literatura actual se ha identificado una relación entre conducta de liderazgo y satisfacción del personal, productividad y compromiso organizacional, aspectos en los que la enfermera administradora desempeña un papel central.

En el mismo contexto del estudio de los estresores en el trabajo, se han realizado comparaciones que documentan altos niveles de estrés en el trabajo comunitario y una prevalencia del burnout en el nivel de atención primaria similar a la registrada en estudios del ámbito hospitalario. ${ }^{26,32}$ Es por ello que hablar de estresores en el trabajo de manera generalizada es insuficiente y destaca la necesidad de que, aun cuando se reconozca que la exposición prolongada culmina consistentemente en síntomas de burnout y serios disturbios fisilógicos $^{27}$, se identifique la naturaleza y el contexto de dichos estresores. En este sentido, en México y en general en el campo, es muy poco lo que se sabe sobre los estresores referidos por enfermeras de primer nivel. Simplemente se ha adoptado la presunción de que el trabajo en este nivel de atención tiene un riesgo bajo; al igual que los hospitales, el primer nivel se ve afectado por el estrés laboral, como lo correspondiente a las características organizacionales, el estilo gerencial de la jefe de enfermeras, la seguridad de la enfermera en el trabajo comunitario y el contacto frecuente con las personas de localidades o colonias en condiciones desfavorables que relatan sus historias a la enfermera. Las múltiples dificultades y frustraciones que enfrenta la enfermera en el campo de la educación para la salud al tratar de que los pacientes modifiquen su conducta ${ }^{33}$. Otros factores que contribuyen al estrés en el primer nivel son las demandas de trabajo, tales como las visitas domiciliarias, los controles administrativos, las interacciones frecuentes con usuarios y pacientes del primer nivel, las relaciones con otras colegas, con médicos, la sensación de dar mucho más de lo que se recibe, la falta de reconocimiento a su trabajo, etc. ¿Cuál es la razón fundamental para excluir el estudio del SB de los ámbitos de primer nivel de atención? Los estudios del SB y las variables organizacionales de mayor impacto en el campo se han observado en el escenario hospitalario a la luz de la prevención de resultados negativos en la salud de los pacientes, sobre todo de los que están en estado crítico. $^{34,35}$ A pesar de ello ¿es esa razón suficiente para considerar que los efectos del SB en los programas de prevención y detección oportuna de las enfermedades son menos importantes? ¿Cuál es el impacto de fallar en los programas de prevención y detección oportuna? ¿Cómo se percibiría esta falla desde la óptica de la salud de la población y la enfermería nacional?

- Además de plantear con claridad los aspectos teóricos subyacentes en cada estudio, es necesario ubicar el nivel de análisis que se busca dentro del modelo propuesto, ya que frecuentemente se observan como resultados de los estudios asociaciones con un sentido poco claro. ¿Se busca el perfil epidemiológico de los diferentes grados de burnout? ¿Se plantean asociaciones causales con diseños inadecuados?

- Una característica fundamental de los estudios nacionales es la ausencia de información sobre los escenarios y variables institucionales en los que se examinó el burnout. Por ejemplo, se habla de sobrecarga de trabajo, pero la cuantificación de la misma depende de la percepción subjetiva de quien la padece. ¿A qué le llamamos sobrecarga de pacientes? Difícilmente se presenta información cuando menos a nivel general de la institución. ¿Cuál es su ocupación en promedio? ¿Se considera insuficiente su plantilla? En caso afirmativo, ¿qué tan insuficiente? ¿Cuál es el coeficiente pacientes/ enfermeras con el que trabajan?

Más allá del dato duro es importante documentar el sistema de organización y de distribución de tareas, el cual varía en función de las distintas unidades de salud e incluso al interior de cada una. En la enfermería nacional, las instituciones de 
salud marcan diferencias aplicables a las diferentes aproximaciones teóricas y niveles de análisis, de forma que se observan diferencias en los salarios, las prestaciones, el tipo de contrato, las políticas de capacitación, los estímulos, etc. Más aún, las diferencias pueden ser más marcadas en hospitales privados, cuyos tamaños y características tienen una mayor variabilidad entre las diferentes empresas.

\section{El SB como efecto y causa dentro de la perspectiva organizacional}

Esta perspectiva en los estudios realizados para los países desarrollados es crucial en la búsqueda de soluciones para reclutar y retener a las enfermeras frente a la carencia actual y proyectada ${ }^{2,36}$. Como se ha planteado anteriormente, la sobrecarga es un componente de las condiciones de trabajo, y la asignación del número de pacientes por enfermera en un servicio u hospital se encuentra inscrita en la organización de la institución, en los problemas de insuficiencia de personal, en la normatividad de las funciones y responsabilidades y en el ambiente laboral, favorable o desfavorable, para el mejor cumplimiento del cuidado del paciente. Al respecto, existe diversos estudios que han analizado la relación entre SB, el coeficiente pacientes/enfermera (como indicador de sobrecarga) y el resultado obtenido en los pacientes. Entre estos estudios sobresalen los realizados en Pensylvania ${ }^{34,35}$, los cuales han documentado el efecto del coeficiente pacientes/enfermera en la mortalidad de pacientes y las fallas en la respuesta a situaciones críticas, así como su relación con la prevalencia de burnout y la satisfac- ción en el trabajo.

Uno de estos estudios incluyó información de 210 hospitales generales, de los cuales se obtuvieron los registros de pacientes y se realizó un seguimiento 30 días después de su egreso para observar mortalidad y reingreso. Los resultados observados entre los pacientes quirúrgicos estudiados fueron los siguientes: un efecto pronunciado del coeficiente pacientes/enfermeras sobre ambos, mortalidad y mortalidad seguida de complicaciones. No obstante el haber controlado el efecto de las características de los pacientes y de los hospitales. Un odd ratio de 1.07 que implica que la mortalidad en los pacientes incrementó un $7 \%$ por cada paciente adicional en el promedio de la carga de trabajo en el hospital. Estos efectos significan que un incremento de personal resultaría en un decremento de mortalidad.

Una de las conclusiones de Aiken $L$ et al. es que las enfermeras forman parte del sistema de vigilancia y seguridad del paciente en un hospital en lo que se refiere a la detección de complicaciones tempranas y una rápida intervención cuando se conoce la condición del paciente, y este sistema se ve influido por el número de enfermeras disponibles y en formación con las que se cuenta para brindar cuidados oportunos, incluyendo la atención de condiciones crónicas. ${ }^{35}$

Por otro lado, Lang et al. ${ }^{37}$, en una revisión sistemática, identificó de entre 490 artículos relacionados con el tema 43 estudios que reunieron los requisitos de inclusión para evaluar el coeficiente pacientes/ enfermeras y su asociación con alguno de los siguientes resultados en los pacientes: falla en el rescate, mortalidad, pneumonía, infección de vías urinarias, úlceras por presión, caídas, errores en la medicación, insatisfacción de pacientes, trombosis venosa, compromiso pulmonar y shock. Los resultados de esta revisión aportaron una mínima información sobre los coeficientes óptimos para unidades de atención aguda. También encontró que no basta con incrementar el número de enfermeras para asegurar la calidad del cuidado o disminuir los efectos sobre diversos indicadores de resultado en la salud del paciente. Finalmente, en esta revisión, el autor no encontró evidencia contundente sobre la relación entre satisfacción en el trabajo y calidad del cuidado.

La enfermería como profesión ha enfrentado la creencia por parte de otros profesionales y de la población de que, para una mejor competencia, la experiencia es más importante que la preparación. Sin embargo, a la luz de los planteamientos antes revisados, se reconocen las características individuales como uno de los componentes de peso en los resultados en las enfermeras y en los pacientes, específicamente las que se relacionan con las habilidades y las destrezas. Aiken et al. ${ }^{38}$ refiere que los beneficios del tipo de formación de las enfermeras en los resultados obtenidos en los pacientes es un aspecto que se ha estudiado poco. La autora analiza dicho efecto en hospitales de Pensilvania con una alta proporción de enfermeras con grado de licenciatura al menos en la atención directa a pacientes. En este estudio se encontró una relación estadísticamente significativa entre la proporción de enfermeras en un hospital con grado de 
licenciatura, de especialización o de maestría y los riesgos de mortalidad y falla en el rescate, controlando las variables sobre los hospitales y los pacientes. Se identificó asimismo que cada $10 \%$ de incremento en el número de enfermeras con el grado más alto de preparación se tradujo en un decremento del riesgo de mortalidad y de fallas en el rescate en una comparación de hospitales con un $20 \%$ versus hospitales con un $60 \%$ de personal con al menos licenciatura. Este trabajo es la primera evidencia empírica que muestra tal asociación y documenta cómo los años de experiencia no fueron predictores de mortalidad en los pacientes de los hospitales estudiados.

Otro aspecto que debe revisarse en el contexto mencionado hasta ahora es la relación entre la sobrecarga de trabajo con el SB y la insatisfacción en el trabajo, así como con los costos de la alta rotación de enfermeras y su reemplazo cuando cambian de trabajo, según los resultados de diferentes estudios ${ }^{4,35}$. Los elementos y las relaciones que forman parte de este aspecto se planten a continuación.

$\mathrm{Al}$ igual que el coeficiente pacientes/enfermera, la prevalencia del SB y su efecto en los resultados en los pacientes han sido ampliamente estudiados en la búsqueda de intervenciones para mejorar la situación de los pacientes en los hospitales en otros países.39 Otra variable que se ha relacionado fuertemente con los resultados en los pacientes, como un factor que agrega estrés o bien que lo media, es la satisfacción en el trabajo. El papel de la insatisfacción en el trabajo ha sido colocado en diferentes lugares en los modelos de investigación utilizados, como causa de desgaste emocional40 y como predictor del burnout41. Asimismo, en términos generales se ha observado una mayor insatisfacción con sobrecargas de trabajo y altos niveles de SB, si bien este aspecto se ha medido con diferentes metodologías. Aun así, el papel mediador de esta variable en la percepción de las cargas de trabajo fue demostrada en una encuesta nacional en Suecia, en la que se observó que mientras más satisfechas estaban las enfermeras con sus trabajos, éstas reportaban menos cargas de trabajo, satisfacción que, desde luego, provenía de variables tales como el nivel de decisión sobre su trabajo. Este aspectos también ha sido planteados por Raiger42, que al considerar el SB desde el lente de la cultura, destaca el componente de los valores. Citando a Flynn y Aiken43, Raiger plantea que, no obstante las diferentes culturas de las naciones, las enfermeras comparten un núcleo de valores globales, entre los que destacan algunos atributos de la práctica tales como la autonomía, el control sobre su ámbito de trabajo y las relaciones con los médicos, como elementos necesarios para sentir satisfacción en el trabajo.

Si bien existe suficiente literatura internacional sobre el tema que nos ocupa, esta revisión deja asuntos pendientes que requieren una mayor exploración, tal es el caso de la utilización del MBI para el estudio de cuidadores, es necesario llevar a cabo una revisión y una reconsideración teórica, determinar si el estudio del SB y el instrumento son aplicables en cuidadores primarios. Es claro que los planteamientos teóricos e instrumento, surgieron del contexto laboral en el que entran en juego los factores organizacionales, fundamentales para la generación del estrés, en este sentido cabe preguntar: ¿Puede efectivamente aplicarse el MBI cuando el cuidador es un familiar del paciente? ¿Existe una correspondencia teórica entre el contexto familiar e institucional?

Otro asunto pendiente es el estudio del burnout desde la perspectiva de género, existen estudios que han analizado los aspectos laborales de las enfermeras con otras variables, fundamental para entender la carga de estrés adicional al laboral. En el contexto nacional, Lara et al.44 plantea que hay algunas variables condicionantes, tales como la edad, la escolaridad, el ingreso y las características del trabajo, que desempeñan un papel frente a las dos situaciones estresantes, la tensión ocupacional y la tensión materna, que pueden desembocar en una de dos alteraciones emocionales posibles, la depresión y la somatización, pero aclara que la presencia de éstas depende de las variables mediadoras, a saber, el conflicto de roles, la identificación de la ideología con la maternidad, la satisfacción por la ayuda recibida de un familiar y la tensión con la pareja. La autora destaca que la tensión ocupacional y la materna tan común en el gremio, se relacionan positivamente con síntomas depresivos y somáticos, sin embargo este aspecto requiere ser discutido en profundidad, ya que en esta revisión queda como asunto pendiente.

\section{Conclusiones \\ Y CONSIDERACIONES FINALES}

Para responder a las preguntas iniciales sobre qué información es necesaria y relevante y sobre cuáles son las aplicaciones específicas en 
el contexto de la enfermería nacional e institucional, sería recomendable, en principio, desarrollar estudios con información comparable (según el instrumento y los puntos de corte para los niveles del SB), sobre la prevalencia del SB en los diferentes ámbitos institucionales y niveles de atención, y con suficiente información sobre las características organizacionales que generan dichas prevalencias y sobre los coeficientes pacientes/enfermeras relacionados con las mismas.

Partir de planteamientos teóricos bien definidos ayudaría a una mejor comprensión del SB, en virtud de la claridad de las relaciones estudiadas y del papel que desempeñan las variables propuestas en los diferentes modelos.

Las respuestas a las preguntas iniciales están sujetas a la preocupante escasez de enfermeras profesionales en el mundo y en nuestro país y al impacto que esta situación tiene en la salud de las enfermeras y en el clima laboral de las instituciones. El problema adquiere una faceta aún más preocupante si además, como la literatura propone, existe una relación entre el agotamiento de los trabajadores y los eventos adversos, los errores en la medicación, el diagnóstico temprano de las complicaciones y la mortalidad de los pacientes en los hospitales45. Por otro lado, la alta demanda por parte de pacientes graves o con enfermedades crónico-degenerativas que requieren de cuidados intensivos o de alta complejidad y la exigencia constante por mejorar la calidad de los servicios de salud hace impostergable la necesidad de profundizar en el estudio de algunos de los factores que condicionan $y / o$ propician el SB.
La investigación del SB debe también considerarse desde la perspectiva del Sistema Nacional de Salud como una vía para la búsqueda de soluciones. En el caso de México, la investigación en enfermería se ha centrado de manera característica en la relación de las variables de asignación de personal, y los hallazgos escasamente se relacionan con los resultados en los pacientes y con factores de la práctica profesional que afectan la seguridad de los mismos y de las enfermeras, tales como el liderazgo y el estilo de gestión, entre otros.

Es indudable entonces que la escasez de enfermeras y su retención en la profesión se ha convertido en una prioridad en nuestro país dada la escasez de las mismas y el creciente número de enfermeras que abandonan la profesión en una etapa temprana46, usualmente como resultado de dificultades en el ambiente de trabajo. Éstas incluyen preocupaciones relacionadas con la poca calidad en el otorgamiento de cuidado seguro, el exceso de horas de trabajo y la consecuente fatiga, y sensación de no ser valoradas o de no ser tomadas en cuenta en la toma de decisiones relacionadas con los pacientes.

Por otro lado, es innegable que las fallas suelen ocurrir cuando el número de enfermeras es insuficiente, cuando están demasiado fatigadas por el exceso de horas de trabajo, o cuando no están bien preparadas en términos de formación o experiencia.

La enfermería en nuestro país y en el mundo enfrenta el reto de otorgar cuidados de calidad y con seguridad para el paciente, sin embargo la dificultad para enfrentar el reto de la escasez de enfermeras obliga a encontrar soluciones que aseguren por un lado la cantidad y calidad adecuada de personal y por el otro los mejores resultados para los pacientes, por lo que desarrollar y fortalecer una línea de investigación sobre el SB de las enfermeras en México, aportará a la construcción de evidencia nacional para la definición de políticas y el diseño de nuevos modelos de organización del sistema de salud que contribuyan a generar mejores condiciones para la práctica de cuidados seguros para los pacientes y así como también para la salud y bienestar de las enfermeras.

\section{Agradecimientos}

Las autoras desean expresar su sincero agradecimiento a la Mtra. María Teresa de Mucha Mora por su valiosa contribución en la revisión del manuscrito.

\section{Referencias BiBLIOGRÁFICAS}

1 Institute of Medicine of the National Academies. Keeping patients safe: Transforming the work environment of nurses. Washington DC: National Academy Press; 2004.

2 CNAC. Creating Healthy Workplace For Canadian Nurses. Final Report of Canadian Nursing Advisory Committee.: Advisory Committe on Health Human Resources; 2002.

3 Smith A, Brice C, Clollins A, Matthews V, McNamara R. The Scale of occupational stress: A further analysis of the impact of demographic factors and type of job: Center for Occupational an Health Psychology School of Psychology, Cardiff University for the Health an Safety Executive; 2000 . 
4 Aiken L, Clarke SP, SLoane DM. Hospital staffing, organization, and quality of care: cross-national findings. Int J Qual Health Care. 2002;14(1):5-13.

5 Aiken L, Clarke S, Sloane D, et al. Nurses' Reports On Hospital Care In Five Countries. Health Aff 2001;20(3):43-53.

6 De los Ríos-Castillo J, Barrios-Santiago P, Ocampo-Mancilla $M$, Ávila-Rojas T. Desgaste profesional en personal de enfermería. Aproximaciones para un debate. Rev Med Inst Mex Seguro Soc. 2007;45(5):493-502.

7 Hernández-Vargas CI, Juárez-García A, Hernández_Mendoza E, Ramírez-Páez JA. Burnout y síntomas somáticos cardiovasculares en enfermeras de una institución de salud en el Distrito Federal. Rev Enfem IMSS. 2005;13(3):125-131.

8 Martínez-López C, López Solanche G. Burnout y síntomas somáticos cardiovasculares en enfermeras de una institución de salud en el Distrito Federal. Arch Med Fam. 2005;7(1):6-9.

9 Cabrera L, López P, Salinas S, Ochoa JG, Marín IA, Haro L. Burnout syndrome among mexican hospital nursery staff. Rev Med Inst Mex Seguro Soc. 2005;43(1):11-15.

10 Rojas A. El estrés laboral en el personal de enfermería. In: Lartigue T, Fernández V, Universidad Iberoamericana Plaza y Valdés, eds. Enfermería: una profesión de alto riesgo. México, D. F.; 1998: 167221.

11 Ortíz L F. Vivir sin estrés. México, D F: Ed. Pax México; 2007.

12 Cordes CL, Dougherty TW. A Review and an Integration of $\mathrm{Re}$ search on Job Burnout. Acad Manage Rev. 1993;18(4):621-656.

13 Garcés de los Fayos E. Burnout: un acercamiento teórico empírico al constructo en contextos deportivos. In. Murcia: Universidad de Murcia; 1994.

14 Maslach C, Schaufeli WB, Leiter MP. Job Burnout. Annu Rev Psychol. 2001;52:397-422.

15 Maslach C, Jackson S. The measurement of experience burnout. Journal of occupational behaviour. 1981;2:99-113.

16 Hingley P. Work-related stress in nursing. Nurs mirror. 2001):1-3.

17 Martín Arribas MC. Estrés relacionado con el trabajo (modelo de demanda-control-apoyo emocional) y alteraciones en la salud: una revisión de la evidencia existente. Enferm Intensiva. 2007;18(4):168181.

18 Agencia Europea para la Seguridad y la Salud en el Trabajo. El estrés relacionado con el trabajo. FACTS 2002;22:1-2.

19 Garcés de los Fayos E. Capitulo 3. Variables predictoras, consecuencias y modelos explicativos del burnout. In: Tesis sobre el burnout; 2003.

20 Atance JC. Aspectos epidemiológicos del síndrome de burnout en personal sanitario. Rev Esp Salud Pública. 1997;71:293-303.

21 Garcés de los Fayos E. Capitulo 2. Instrumentos de medida del burnout y aspectos metodológicos asociados. In: Tesis del Burnout 2003.

22 Gil-Monte P. Validez factorial de la adaptación al español del Maslach Burnout Inventory General Survey. Salud Publica Mex. 2002;44(1):3340.

23 Langballe EM, Falkum E, Innstrand ST, Aasland OG. The Factorial Validity of the Maslach burnout Inventory-General Survey in Representative Samples of Eight
Different Occupational Groups. Journal of career Assessment. 2006;14(3):370-384.

24 Gil-Monte P, Peiró JM. Perspectivas teóricas y modelos interpretativos para el estudio del síndrome de quemarse por el trabajo. Anales de Psicología. 1999;15(2):261268.

25 Gershon R, Stone P, Zeltser M, Faucett J, Macdavitt K, Chou SS. Organizational Climate and Nurse Health Outcomes in the United States: A Systemic Review. Ind Health. 2007;45(622-636).

26 McGrath A, Reid N, Boore J. Ocupational Stress in Nursing. Int J Nurs Stud. 1989;26(4):343-358.

27 Mc Vicar A. Workplace stress in nursing: a literature review. J Adv Nurs. 2003;44(6):633-642.

28 Poncet M, P T, Papazian L, et al. Burnout Syndrome in Critical Care Nursing Staff. Am J Respir Crit Care Med. 2007;175:698-704.

29 Ríos RMI, Godoy FC, Peñalver $\mathrm{HF}$, et al. Estudio comparativo del burnout en personal de enfermería de Cuidados Intensivos y de Urgencias. Enferm Intensiva. 2008;19(1):2-13.

30 Maldonado DM, Morales KM. EL personal de enfermería y la unidad de cuidados intensivos neonatales. In: Lartigue T, Fernández V, Universidad Iberoamericana Plaza y Valdés, eds. Enfermería: una profesión del alto riesgo. México, D. F.; 1998: 311-343.

31 Shirey MR. Stress and Coping in Nurse Managers: Two Decades of Research. Nurs Econ. 2006;24(4):193211.

32 Soto R, Santamaría M. Prevalencia del burnout en la enfermería de atención primaria. Enferm Clin. 2005;15(3):123-130.

33 Bimbela JL, Gorrotxategi M. Ha- 